\title{
MELA: Monitoring and Analyzing Elasticity of Cloud Services
}

\author{
Daniel Moldovan, Georgiana Copil, Hong-Linh Truong, Schahram Dustdar \\ Distributed Systems Group, Vienna University of Technology \\ E-mail: \{d.moldovan, e.copil, truong, dustdar\}@dsg.tuwien.ac.at
}

\begin{abstract}
Cloud computing has enabled a wide array of applications to be exposed as elastic cloud services. While the number of such services has rapidly increased, there is a lack of techniques for supporting cross-layered multi-level monitoring and analysis of elastic service behavior. In this paper we introduce novel concepts, namely elasticity space and elasticity pathway, for understanding elasticity of cloud services, and techniques for monitoring and evaluating them. We present MELA, a customizable framework, which enables service providers and developers to analyze cross-layered, multi-level elasticity of cloud services, from the whole cloud service to service units, based on service structure dependencies. Besides support for real-time elasticity analysis of cloud service behavior, MELA provides several customizable features for extracting functions and patterns that characterize that behavior. To illustrate the usefulness of MELA, we conduct several experiments with a realistic data-as-a-service in an M2M cloud platform.
\end{abstract}

Keywords-elastic computing, cloud service, elasticity monitoring, elasticity analysis

\section{INTRODUCTION}

With the increasing popularity of cloud systems, the number of applications and systems born in or migrated to cloud environments has substantially increased. Diverse types of stakeholders in cloud environments, ranging from business oriented software providers to e-science professionals, have continuously investigated techniques exposing diverse functionalities as a service (XaaS). In this context, substantial effort have been paid for the development of emerging elastic cloud services, which scale up/out as long as the workload is high, and scale back in/down when possible, reducing cost while maintaining performance and quality. Going beyond the traditional "elastic scalability" which concentrates on scaling in/out resources to achieve performance, in general, elastic cloud services have three main dimensions: "resource elasticity", "cost elasticity", and "quality elasticity" [1].

Developing and managing elastic cloud services with such multi-dimensional elasticity is challenging. One of the main questions is how to monitor and evaluate cloud service's elastic behavior, determining the proper cost and quality indicators and their boundaries, and utilize them for optimizing and controlling the services' elasticity. Currently, deciding cost and quality indicators and their boundaries is a difficult task, usually done by cloud experts. Existing monitoring and analysis tools focus either on the whole cloud service level [2] or on

This work was partially supported by the European Commission in terms of the CELAR FP7 project (FP7-ICT-2011-8 \#317790) the underlying virtual infrastructure [3][4], and do not provide a cross-layered, multi-level service elasticity behavior picture, hindering the discovery of the cause for stakeholder goal's violations. For controlling elastic cloud services one must be able to detect if a goal violation originates in a poorly chosen cloud service, subscription scheme, resource congestion, or failing service unit. In particular, we think that in order to understand elastic cloud services, we need to investigate new concepts that can be used to characterize the cloud service's elastic behavior from multi-dimensional monitoring data.

In this paper, we present MELA, a framework for monitoring and analyzing elasticity of cloud services. MELA allows cloud service developers and providers to trace their service behavior from the whole service level to the underlying virtual infrastructure, extracting characteristics and providing crucial insight in their elastic behavior. Providing aggregated monitoring data and analysis of elastic cloud services, MELA acts as a base for analyzing and controlling elastic cloud services. The main contributions of our paper are:

- a novel concept of elasticity space and elasticity pathway for analyzing elastic behavior of cloud services at multiple levels.

- customizable mechanisms for extracting runtime boundaries of cloud service's elasticity that fulfill user-defined elasticity requirements.

- techniques for evaluating multi-level user-defined elasticity requirements over service's cost, quality, and resources, analyzing the elastic behavior of cloud services.

The rest of this paper is structured as follows. Section II presents the motivation and research problems. Section III presents our techniques for monitoring and analyzing elasticity of cloud services using the elasticity space and pathway. In Section IV we describe the MELA framework. Section V presents our prototype and experiments. We discuss related work in Section VI. Section VII concludes the paper and outlines the future work.

\section{MOtIVATION \& RESEARCH PROBLEMS}

Let us consider a realistic data-as-a-service (DaaS) application for an M2M cloud platform-as-a-service, for which we have user-defined elasticity requirements ${ }^{1}$ w.r.t service runtime performance and cost.

\footnotetext{
${ }^{1}$ In this paper "users" refer to the users of our framework: cloud service provider, developer and elasticity controllers.
} 


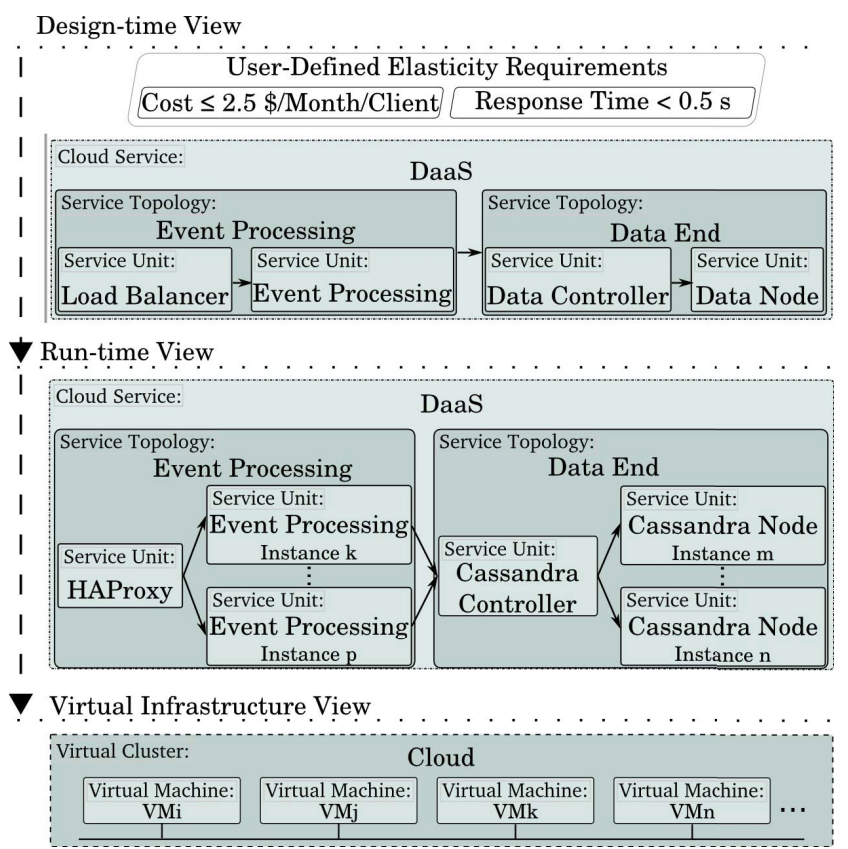

Fig. 1: Elastic Cloud Service Views

In general, we have three main views from which cloud services are described (Fig. 1): (i) design-time view, where we see the whole service dependency model (Cloud Service, Service Topology, Service Unit) and user-defined service requirements, (ii) run-time view, where instances of several Service Units are deployed and executed in virtual machines, and (iii) the virtual infrastructure, where several virtual machines, possibly grouped in virtual clusters, are used. From the design-time point of view, in this case the DaaS has two service topologies, Data End and Event Processing, supporting horizontal scaling by addition and removal of VMs. The Data End service topology includes two service units, a Data Node holding data, and a Data Controller managing it. The Event Processing service topology also contains two service units: Load Balancer, distributing client requests, and Event Processing interacting with the Data End. At run-time, the Data End units uses Cassandra ${ }^{2}$ for its service units, and the Event Processing uses HAProxy ${ }^{3}$ for its Load Balancer service unit. Moreover, at run-time, due to user-defined elasticity requirements, service unit instances are added/removed dynamically, triggering allocation and deallocation of virtual machines at the virtual infrastructure level. Thus, Cassandra was chosen as it supports adding/removing of data nodes without restarting the cluster controller (any such data store would have sufficed), and HAProxy is used as it also supports transparent addition/removal of web servers hosting Event Processing service units.

User-defined service requirements can be viewed as elasticity requirements, as they restrict the elastic behavior of the

\footnotetext{
${ }^{2}$ http://cassandra.apache.org/

${ }^{3}$ http://haproxy.1wt.eu/
}

DaaS. To enforce such restrictions at the virtual infrastructure level, i.e. when to add/remove a VM, of what type, under which pricing scheme, the restrictions need to be linked and mapped to the run-time view. Monitoring data from the run-time view must also be linked back to the user-defined elasticity requirements. This enables the discovery of service units belonging to service topologies that cause requirements violations. For example, using MELA, one should be able to determine that a high service cost is caused by too few clients served at the Event Processing service topology level because of overloaded Event Processing service unit instances, or high communication latency between the Event Processing instances and the Data Controller.

While using various monitoring techniques, such as [4] or [5], we can capture monitoring data from the whole service level or virtual infrastructure level, such data typically does not answer the following crucial questions:

- what should be the behavior of the service topologies and units when fulfilling user-defined elasticity requirements.

- when is the service behavior elastic, i.e. adapting and fulfilling user-defined elasticity requirements.

- what is the cause of an elasticity requirement violation, traced from the design-time view to the underlying virtual infrastructure.

- how does the service's elastic behavior evolve in time, i.e. what are the correlations and patterns in its behavior.

Capturing, describing and analyzing elastic behavior of cloud service is crucial not only for developers who build and optimize cloud services, but also for software controllers that change the topology of such services at run-time, enforcing user-defined elasticity requirements. Such controllers need a monitoring and analysis mechanism that extracts elasticity characteristics, which can be used to refine user-defined elasticity requirements or predict the service behavior, leading to better service control and quality.

This motivates us to investigate the following issues:

- Which concepts can be used to capture the elastic behavior of cloud services?

- How to extract characteristics that describe the service's elastic behavior to support both reactive and predictive control of elastic services?

- How to analyze the cloud service's behavior, detecting the source of user-defined elasticity requirements violations?

This paper focuses on capturing the properties of elastic services at multiple levels, providing support for analyzing their behavior from multiple views, and characterizing the elastic behavior of each cloud service based on user-defined elasticity requirements.

\section{Monitoring And Analyzing Elastic Cloud SERVICES}

\section{A. Runtime Properties of Elasticity of Cloud Services}

In order to support multi-dimensional analysis of elastic services behavior [1], we categorize monitoring data in three dimensions: Cost, Quality, and Resource. Each dimension 


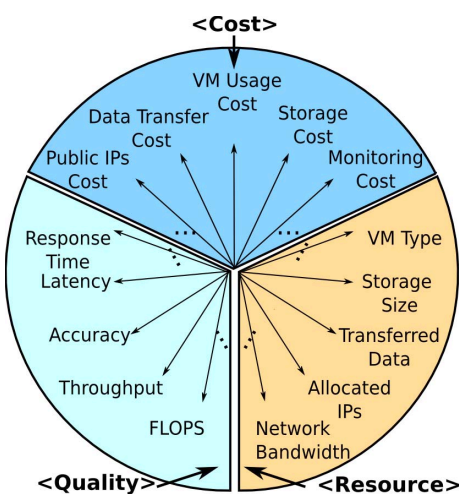

Fig. 2: Elasticity Dimensions

has a set of metrics, shown in Figure 2. These categories are sufficient for capturing low-level data about any monitored element (e.q., service topology or service unit) within a cloud service that can be used for understanding the elastic behavior of that service. Conceptually, to capture monitoring data associated with a monitored element at a specific time $t$, we define the monitoring snapshot, ms:

$$
m s=\left(\left\langle c_{i}\right\rangle,\left\langle q_{j}\right\rangle,\left\langle r_{k}\right\rangle, t\right)
$$

where $c_{i} \in$ Cost, $q_{j} \in$ Quality, and $r_{k} \in$ Resource.

Monitoring snapshots capture metrics, but do not provide information about boundaries over the metric's values in which user-defined elasticity requirements are fulfilled. Therefore, in order to analyze the elastic behavior of a monitored element, we represent metric boundaries extracted from user-defined elasticity requirements as well as boundaries detected/evaluated by our techniques using the elasticity boundary:

Definition 1. An elasticity boundary describes the upper and lower bound over a set of metrics for a monitored element.

Conceptually, an elasticity boundary elBoundary is:

$$
\text { elBoundary } \left.\left.\left.=\left(\left\langle c_{i}^{u}, c_{i}^{l}\right)\right\rangle,\left\langle q_{j}^{u}, q_{j}^{l}\right)\right\rangle,\left\langle r_{k}^{u}, r_{k}^{l}\right)\right\rangle\right)
$$

where $c_{i}^{u}$ and $c_{i}^{l}$ denote the upper bound and the lower bound of metric $c_{i}$, respectively, $q_{j}^{u}$ and $q_{j}^{l}$ for $q_{j}$, and $r_{k}^{u}$ and $r_{k}^{l}$ for $r_{i}$. All metrics $c_{i}, g_{j}, r_{k}$ are specified in elasticity requirements indicating the parameters under which the cloud service is elastic. In the rest of this paper, user-defined elasticity boundary and evaluated elasticity boundary are used to indicate the boundary extracted from user-defined requirements and determined from elasticity monitoring, respectively.

\section{B. Elasticity Space and Pathway}

Given a set of monitoring snapshots and a user-defined elasticity boundary, we need to understand when a monitored element is in elastic behavior, if its behavior violates the elasticity boundary and if we can characterize the service behavior using some specific "pathways". Naturally, we expect that the meaning of "elasticity" will be dependent on the types of monitored elements, their runtime settings and requirements.
To this end, we define the concept of elasticity space to determine if a monitored element is in elastic behavior:

Definition 2. An elasticity space captures all runtime metrics described in the user-defined elasticity boundary when a monitored element is in elastic behavior, which is determined via an elasticity space function.

Formally, let $f_{\text {elSpace }}$ be an elasticity space function, $M S=$ $\left\{m s_{i}\right\}$ be the set of monitoring snapshots, then an elasticity space elSpace can be defined as: elSpace $=f_{\text {elSpace }}(M S)$.

A $f_{\text {elSpace }}$ has to perform two steps: detect when an elastic behavior starts and stops, and extract only monitoring data describing the service behavior while respecting the userdefined elasticity boundaries. In principle, there could be several elasticity space functions, which can be developed for and applied to different types of monitored elements, such as specific types of service units, service topologies, or the whole cloud service. Furthermore, these functions are also dependent on the metrics in user-defined elasticity boundaries.

An elasticity space function is designed to extract useful information about the overall behavior of the cloud service when elasticity requirements are fulfilled. A space would contain, for example, only the throughput and $\operatorname{cost} / V M / h$ metrics from which the serviceCost/client/h targeted by requirements can be determined, not including metrics that have no impact on it. One can analyze the behavior of an elastic cloud service by checking if its elasticity space is within the user-defined elasticity boundaries.

While the elasticity space enables cloud service elasticity analysis, it does not provide insight into relationships between metrics influencing the elastic behavior over time, e.g., throughput and cost $/ V M / h$ might or might not follow a linear relationship. In order to characterize the elastic behavior from specific views/perspectives over a cloud service, we define the concept of elasticity pathway.

Definition 3. Given a specific view on metrics $V=$ $\left\{m_{1}, m_{2}, \cdots, m_{n}\right\}$, an elasticity pathway for $V$ characterizes the elasticity relationship among $m_{i}$ over the time.

Formally, an elasticity pathway elPtw is determined by a function $f_{\text {elPtw }}$ which takes as input an elasticity space elSpace and a view $V$ over the space's metrics, and returns another function describing behavioral patterns or characteristics of the the monitored element: elPtw $=g(V)=$ $f_{\text {elPtw }}($ elSpace,$V)$. Various elasticity pathway functions can be defined over the elasticity space, enabling space analysis from multiple perspectives. An elasticity pathway function is designed to perform a complex evaluation of the cloud service behavior, determining characteristics that can be used to predict the service's behavior. Such a function could extract metric correlations, e.g., between throughput and $\operatorname{cost} / V M / h$ for a service unit, determining the influence of throughput on the cost. As the elasticity pathway function is applied over a elasticity space, the quality of the determined elasticity pathway being heavily influenced by the size and data in the elasticity space. 


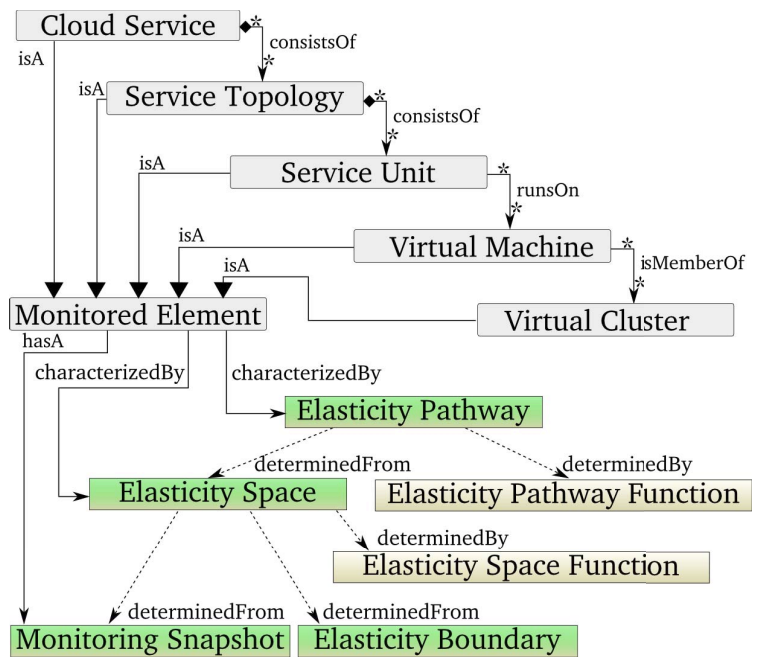

Fig. 3: Association between Elasticity Space/Pathway and Monitored Elements

\section{Multi-Level Elasticity Space of Cloud Services}

In order to monitor and evaluate the elasticity space and pathways of a cloud service, we need to (i) build the cloud service dependency model, (ii) build the monitoring snapshot for all monitored elements, and (iii) apply particular elasticity space and pathway functions over the monitoring snapshots.

1) Elastic Cloud Service Dependency Model: For describing elastic services from the whole service level to the underlying virtual infrastructure, we need an abstract representation model that enables the decomposition of user-defined elasticity requirements in lower level requirements which can be mapped to the virtual infrastructure. To support analysis of service behavior at different levels, we represent a cloud service composed of service topologies, each topology containing several service units, which are deployed on one virtual machines belonging to virtual clusters (Figure 3). Based on the dependency model, we build composite monitoring snapshots which aggregate metrics bottom-up, creating based on lower level metrics higher level ones which can be targeted by userdefined elasticity requirements. At different levels we allow the association of different elasticity space and pathway functions, enabling multi-level custom analysis of cloud service behavior. Using the dependency model we combine monitoring snapshots, elasticity spaces and pathways from one level and propagate them to the other levels, obtaining a complete view over the cloud service behavior.

Listing 1: Example of M2M DaaS Dependency Model

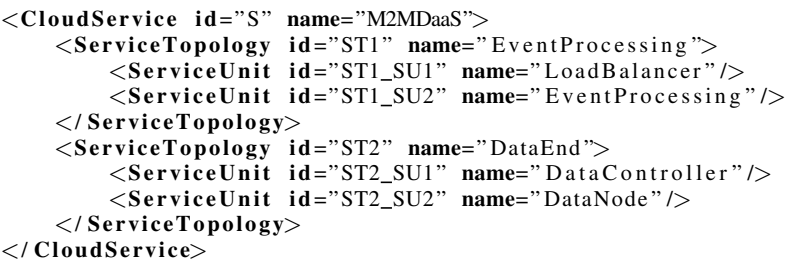

To build the elasticity space, the dependency model of the cloud service is extracted or defined by users/tools, and used for analyzing the service behavior. For easy integration with other tools, we use an XML-based representation. Listing 1 shows the M2M DaaS containing two service topologies and their service units.

2) Cross-layered Metric Composition: Monitoring snapshots capture (low-level) metrics, but not necessary the same as elasticity requirements defined by the user. For example, a monitoring snapshot for the Event Processing service unit might only include responseTime, throughput and $\operatorname{cost} / V M / h$ while the elasticity requirements might target serviceCost/client/h and numberOfClients. Therefore, to obtain a complete view over the cloud service behavior, from low level metrics to higher ones, we develop techniques for cross-layer composition of monitoring snapshots, elasticity spaces and elasticity pathways, following the cloud service structure. This composition introduces the problem of combining/aggregating metrics. Depending on the type of metrics, a valid composition of two metrics might involve different operations. We define an XML-based domain-specific language for describing such metric composition rules as a cascading sequence of operations which apply one or more operators over one or more operands. Defining the composition rules requires domain specific knowledge, e.g., in determining that the cost of a service unit is computed from the hourly cost per VM instance and the network data transfer.

Based on the metric composition, we provide an analysis mechanism for determining the elasticity space and pathway at different dependency model levels, providing a multi-level decomposition of the cloud service behavior. Such a decomposition is beneficial to service controllers, which can use our approach to detect which monitored element from which service dependency model level violates service requirements, and reason in terms of metrics located at that particular level. Providing a complete view over the behavior of cloud services enables service provider/developer and software controllers to reason on the same service using separate perspectives.

In Listing 2 we show a composition rule obtaining the cost of data transfered per client per hour for the Data End service topology. The metric is obtained by summing up the values of the dataOut metric from all service topology children having level service unit, and dividing the result with

Listing 2: Example of Metric Composition

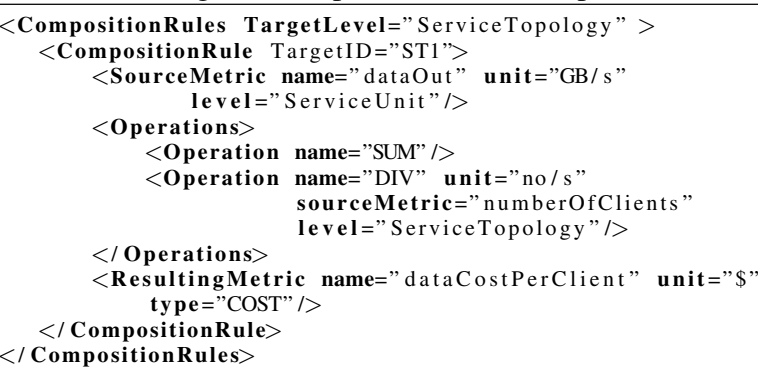




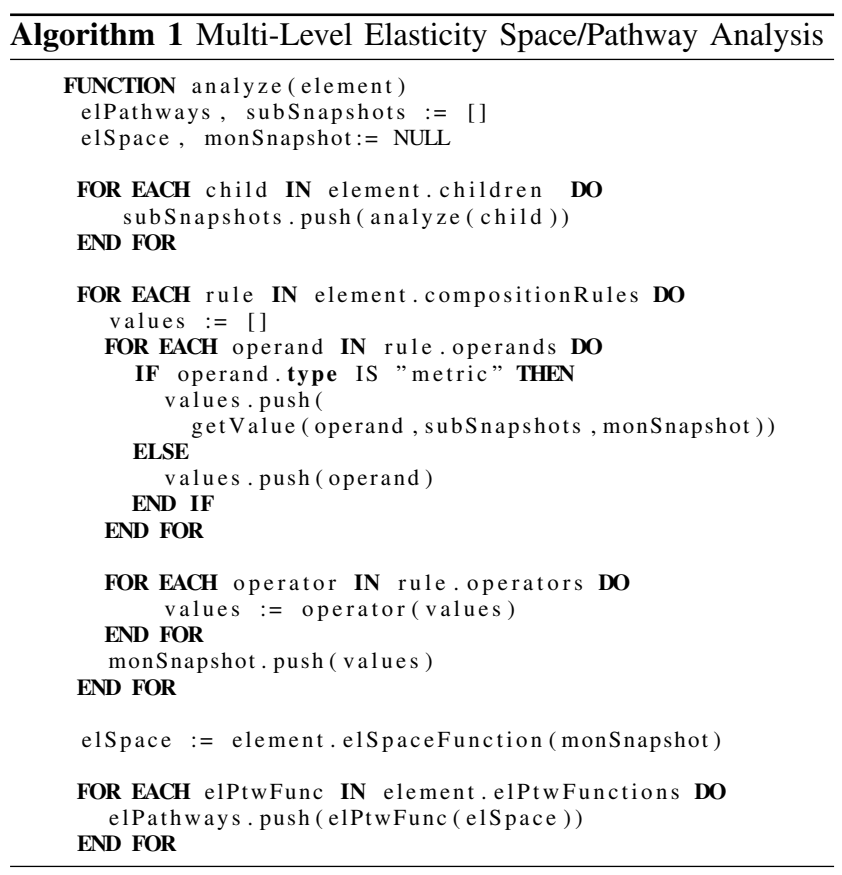

the value of the numberOfClients metric retrieved from the target service topology.

3) Multi-level Elasticity Space/Pathway Analysis: Algorithm 1 traverses the the service dependency model in a depth-first manner and carries out runtime analysis. For every element at each level, its metric composition rules are applied, building a multi-level monitoring snapshot. Each composition rule contains a set of operands (metrics or values) and a set of operations from $\{$ sum, max, min, avg, div, add, sub, mul, concat, union, set, keep\}. Over the space we apply the elasticity pathway functions, analyzing the cloud service behavior.

\section{Elasticity Monitoring as a Service}

\section{A. MELA Overview}

Based on our concepts in Section III, we develop MELA, elasticity monitoring and analysis as a service (Fig. 4). MELA contains a core MELA Service, and Data Collector nodes. A Data Collector node is a customizable component that gathers from existing monitoring solutions data associated with a dependency model level or monitored element (e.g., responseTime or throughput for the Event Processing service topology), and sends it for processing and analysis to the MELA Service.

Monitoring data from existing monitoring systems is usually associated with a single level, e.g., virtual infrastructure, service topology or service unit. An important MELA feature is the linking of these levels, which implies a configuration step using the Elasticity Functions Management API, defining for the monitored elements at each level the composition operations to be applied. This step feeds data into the Elasticity Pathway \& Elasticity Space Functions repository which also

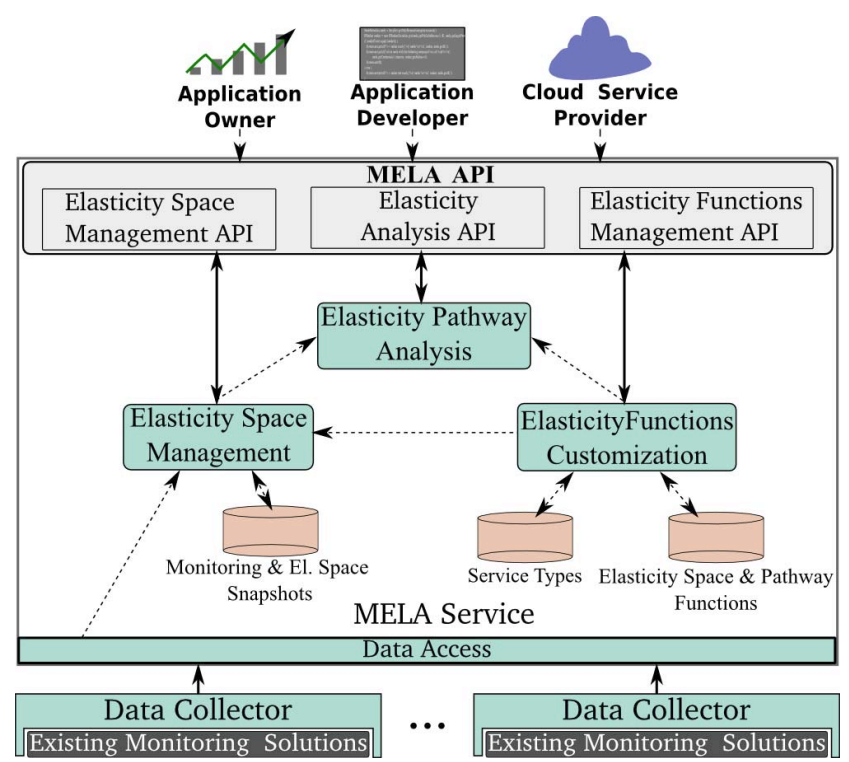

Fig. 4: MELA Overview

contains the composition rules for building the monitoring snapshot. As the elasticity space is determined from monitoring snapshots, the Elasticity Space Management unit also handles the monitoring snapshot construction and stores them in the Monitoring \& Elasticity Space Snapshots database.

MELA exposes its functionality through REST services and Java API, providing methods for configuring MELA and analyzing elastic service behavior.

\section{B. Elasticity Space and Pathway Function Prototypes}

In the current MELA prototype, we implement an elasticity space function which, starting from user-defined elasticity requirements for the whole cloud service, determines as space boundaries for all service topology and service unit instances, their maximum and minimum encountered metric values when the user-defined elasticity requirements are respected.

Based on the elasticity space, custom elasticity pathway functions can be defined as MELA plug-ins, enabling custom analysis of service behavior. For the prototype elasticity pathway function, we adapt an unsupervised behavior learning technique using self organizing maps (SOMs)[6], and classify monitoring snapshots by encountering rate in DOMINANT, NON-DOMINANT, and RARE. Such a pathway is important for understanding if the regular behavior of the service respects user-defined elasticity requirements.

As SOMs are unsupervised neural networks that map multidimensional spaces into low dimensional ones, we use them for grouping monitoring snapshots. Each SOM's neuron value is derived from its snapshots. Each monitoring snapshot is mapped to the group from which it has the smallest distance. With each new snapshot, the group and its SOM neighbors are updated using the function $V_{\text {new }}($ group $)=V_{\text {old }}($ group $)+$ $A * N($ group $)\left(V(\right.$ snapshot $)-V_{\text {old }}($ group $\left.)\right)$, where $A$ is a discount factor, and $N$ (group) is a neighborhood function 


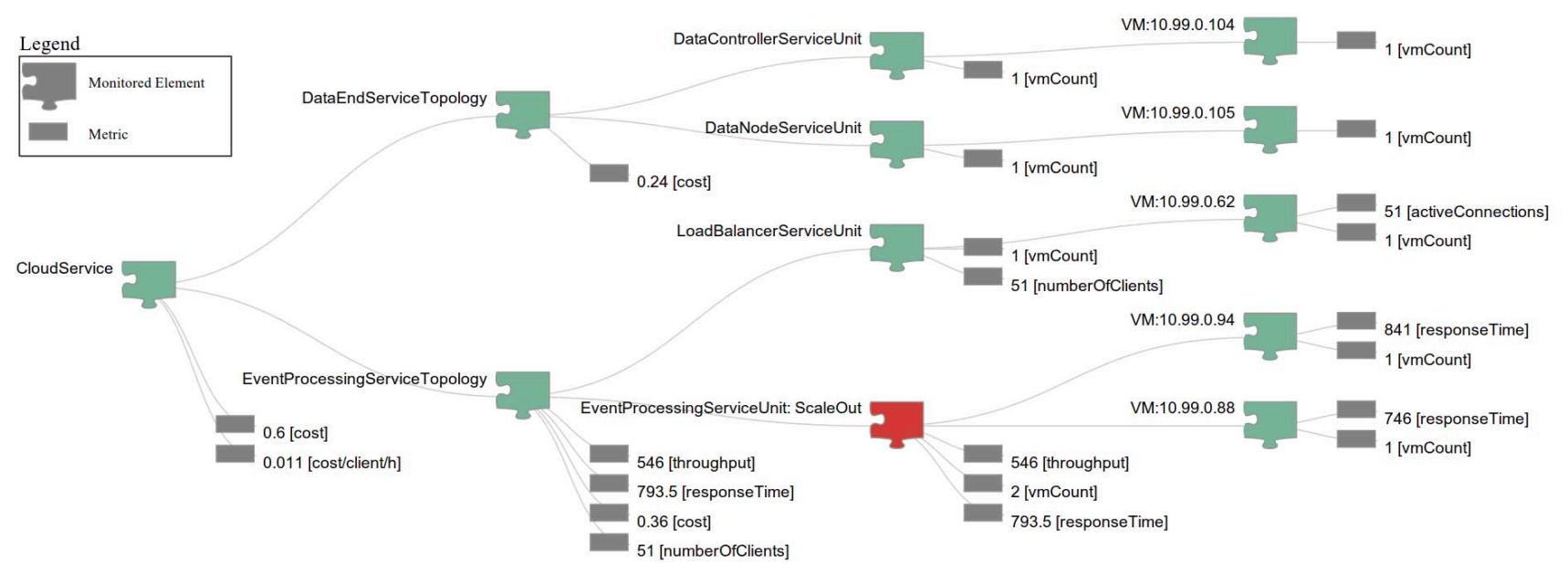

Fig. 5: MELA Visualization of Multi-Level Monitoring Data

determining the degree with which a group value is updated. We initialize the SOM with snapshot groups having all metrics equal to 0 , and rely on its self-adaptive nature to map the input data. We use a neighborhood function of 1 for the directly targeted group and of 1/neighbourLevel/neighboursCount for the group neighbors. Updating the neighbors creates and update new groups, mapping the input data better. The discount learning factor is $1 /$ neighbour Level, the neighborhood is 2 and the map size is $10 \times 10$. A filtering step merges groups with same value, consolidating the monitored snapshots.

\section{EXPERIMENTS}

We apply MELA ${ }^{4}$ to monitor and analyze the realistic data-as-a-service application for an M2M cloud mentioned in Section II. From real sensor data, we simulated a Gaussian distribution of M2M sensors - the clients connected to our system, starting from 50 sensors per hour, increasing up to 350 , and then decreasing again. Each request from a sensor will require between 1 and 10 operations on the data service. For practical reasons we simulated a hour of experiment each second. The service VMs were deployed on our OpenStack ${ }^{5}$ cloud. For these experiments MELA utilizes Ganglia ${ }^{6}$ as base for the Data Collector nodes, retrieving generic OS level and service specific monitoring data (e.g., clients/h, throughput, response time) from custom Ganglia plug-ins.

\section{A. Monitoring Elasticity Space}

We start from the scenario in which the cloud service provider wants to monitor and define elasticity requirements for the service cost and performance. Using our cross-layered metric composition mechanism, we derive service level metrics from low level ones. We start at the Service Topology level defining the cost based on vmCount (number of service

\footnotetext{
${ }^{4}$ Detailed MELA API, prototype implementation, and experiments can be found at http://dsg.tuwien.ac.at/research/viecom/mela/

${ }^{5}$ http://www.openstack.org/

${ }^{6} \mathrm{http} / / /$ ganglia.sourceforge.net/
}

unit instances) and the cost per virtual machine (assumed 0.12 EUR per machine per hour). Summing up at the Service Level the cost obtained from the Service Topology level, we obtain estimated hourly service cost. To monitor the service performance, we define multiple metrics, averaging the number of service clients/ $h$ (active connections), responseTime and throughput at every Service Unit level and propagating them to the upper service dependency model levels. For monitoring the service cost efficiency, a metric is defined at service level, dividing the cost by the value of the clients/ $h$ metric, obtaining the estimated cost/client/h. Figure 5 presents the MELA crosslayer metric monitoring snapshot obtained from composing and propagating low level metrics. Obtaining higher-level composite metrics, the service controller can define userdefined elasticity requirements on them, analyze if they are fulfilled, and, if not, enforce them by adding/removing VMs to/from service unit instances.

\section{B. Elasticity Space Analysis}

MELA's second feature is elasticity space analysis, which determines what are the behavioral boundaries in which the service fulfills supplied requirements. Continuing with the previous scenario, the cloud service provider wants to implement a $2.5 \$$ monthly subscription for each service client (sensor). Using MELA, the provider can monitor the service elasticity behavior (via metrics) at each service level, to guarantee he/she does not end up paying more than $2.5 \$$ per month per client to the cloud provider. Assuming a month of 30 days each with 24 hours, the elasticity requirement is a cost of at most $0.0034 \$$ per served client per hour. Using our elasticity space function prototype, we determined the elasticity space, including the maximum and minimum encountered values for the service metrics in which the service requirements are fulfilled, for each service level and monitored element.

From the snapshot of the elasticity space for the Event Processing Service Topology (Fig. 6a), one can examine how individual metrics evolve over the time. For example, 


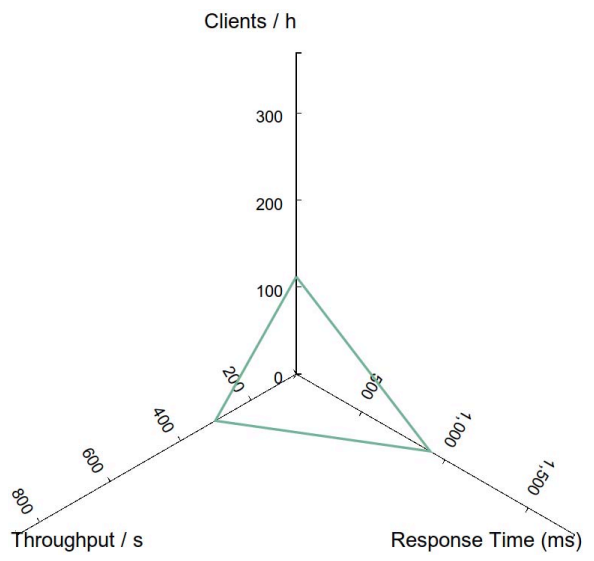

(a) Elasticity Space Snapshot

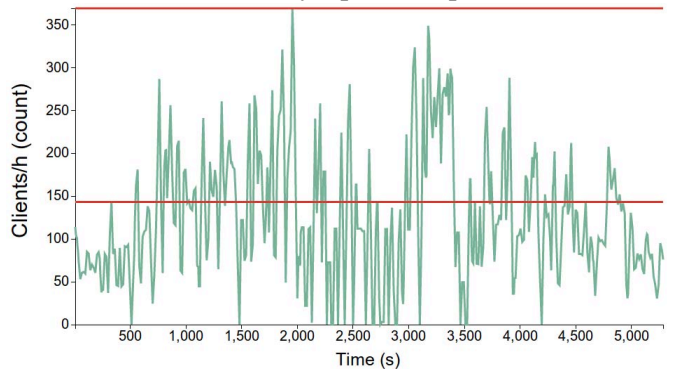

(b) Elasticity Space "clients/h" Dimension

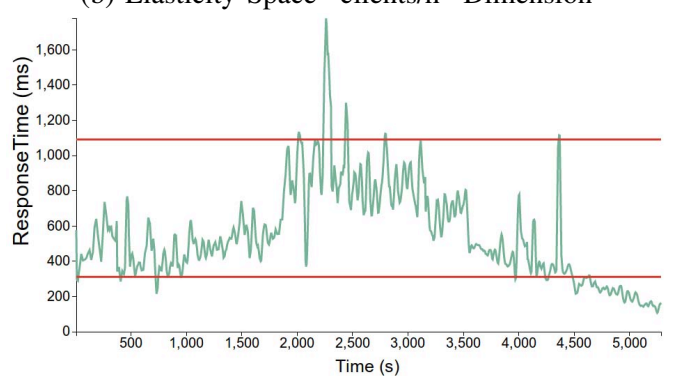

(c) Elasticity Space "responseTime" Dimension

Fig. 6: Elasticity Space for Event Processing Service Topology

we analyze the elasticity space clients/h dimension, marking with flat lines the minimum and maximum acceptable values (Fig. 6b). The clients/h minimum is 172 , which multiplied by 0.0034 , gives a $0.48 \$$ per hour, indicating that the service uses at least $4 \mathrm{VMs}$ at $0.12 \$$ per hour. From this evaluated elasticity boundary we can deduce that given the current service control strategy, there should be at least 172 clients per hour to justify the cost of running in cloud. To learn more about the service behavior and the boundaries the service controller should enforce on different levels, boundaries are also extracted for the responseTime describing the time to process a request, and throughput which describes the number of operations performed by the service per second (a client can perform many operations). Focusing on responseTime (Fig. 6c), MELA extracts a minimum boundary, which might seem weird at first, as we would expect to always want minimum response time. In

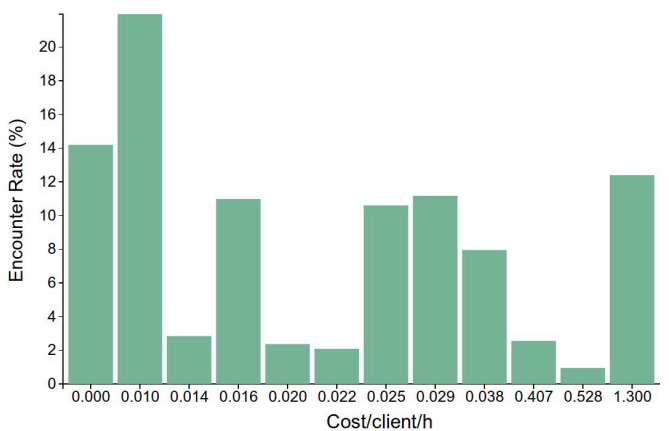

Fig. 7: Elasticity Pathway of CloudService cost/client/h

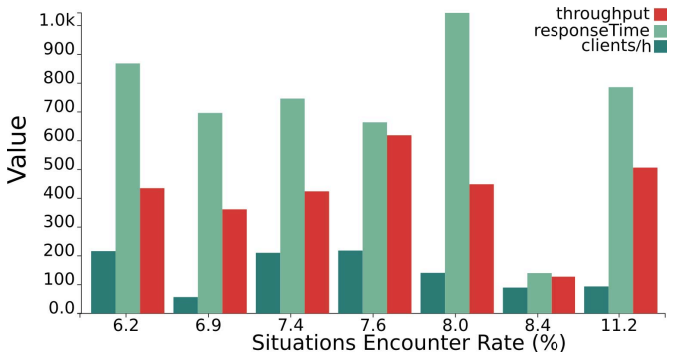

Fig. 8: Dominant Elasticity Pathway of Event Processing Service Topology

this case, this evaluated elasticity boundary tells us that a too low response time means that the service has underused virtual machines, which is not cost effective. This conclusion can be reached by further investigating the elasticity boundaries for the rest of the service levels, which we do not expand here due to lack of space.

Continuing with the scenario, knowing the boundaries for different service levels and monitored elements, the cloud service provider wants to extract service characteristics that indicate if the usual service behavior is within the determined space boundaries. Towards this we apply our elasticity pathway function prototype which groups combination of different metric values as DOMINANT, NON-DOMINANT, and RARE, according to the rate at which they are encountered in the monitored data. Such a classification highlights the usual behavior of the service and the correlations between the analyzed metrics. Applying the pathway on the cost/client/h cloud service level metric (Fig. 7), from the encounter rates and the metric values, it is visible that the cost/client/h is less than $0.0034 \$$ only in approximate $76 \%$ of the encountered situations, leading to the conclusion that a pricing scheme of $2.5 \$$ per month per client is not fully sustainable. To find out why, the provider uses the MELA multi-level analysis feature to focus on the Event Processing Service Topology. The elasticity pathway is applied on the clients/h, responseTime, and throughput (operations) metrics, the metrics influencing the service performance, and thus, the number of served clients and the service cost efficiency. From the DOMINANT behavior (Fig. 8), it is visible that by summing 
up the encounter rates, in approximate $35 \%$ of the encountered situations, the service had less than the required 172 clients. Analyzing further, only in $8 \%$ of these situations we detect few clients with low throughput, while in the rest $27 \%$ we detect few clients but high response times and throughput, indicating a potential performance bottleneck. To determine the correct cause of the high cost per client, the cloud service provider needs to further investigate the elasticity space and pathways of the other monitored elements, from topology level information such as latency or cost for the Data Service Topology, to the service units, and low level performance (CPU usage, memory usage, latency) of associated VMs.

This section has showcased the MELA features of multilevel metric composition and service behavior analysis, and highlighted the importance to monitor and analyze the behavior of elastic services at multiple levels.

\section{RELATED WORK}

A scalable framework for data collection and aggregation is introduced in [3]. Our tool differs as it links service level to virtual infrastructure level monitoring data, providing a complete view over the monitored cloud service behavior. An architecture for dynamically controlling the behavior of cloud services using a set of high-level rules is introduces in [7]. Combining such rule-based systems with MELA would enable the specification of more powerful rules, that target higher level metrics (at the service unit, topology or whole service level), providing multi-level service control. The cost of Amazon EC2 spot instances is analyzed in [8], and [9] discusses cost-effective strategies for using such instances. We differ as we provide a mechanism for extracting cost elasticity boundaries, understanding limits for the cost behavior of elastic cloud services.

The authors of [10] improve existing monitoring systems using custom metric aggregation scripts and service model information, while we provide a customizable mechanism for mapping metrics to the service model and deriving higher level information. A mechanism for adapting cloud allocation is presented in [2], using an aggregator that monitors the workload at each service tier, while we employ a generic approach that can analyze a wide array of services. In [11] the authors monitor cloud resource usage for the infrastructure owner, while we adopt the cloud infrastructure user perspective. In [5] and [12] the authors comprehensive monitoring systems collecting both virtual infrastructure and service level information, while we structure and focus on understanding the dependencies between the monitored metrics. Monitoring performance and data delivery is also the focus of [13], presenting a solution tailored for a specific virtualization framework. An elastic monitoring framework for cloud infrastructures is presented in [14], providing a powerful query mechanism for retrieving service level information. In our approach we also enrich monitoring data with derived metrics, and perform service behavior analysis towards extracting elasticity characteristics.

\section{CONCLUSIONS AND FUTURE WORK}

To support the monitoring and analysis of elasticity of cloud services, this paper introduced elasticity space and elasticity pathway as novel concepts characterizing behavior of elastic cloud services. We have presented MELA which supports realtime multi-level analysis of elastic cloud services. MELA also provides several features for customizing and integrating different elasticity analysis functions to support the analysis of other complex elastic behaviors.

For the future we will enhance our prototype by incorporating cloud infrastructure elasticity analysis with our elastic cloud service analysis. We will enhance MELA with pattern based elasticity space and pathway functions. Furthermore, we will work on automatic extraction of elasticity dimensions dependencies and automatic discovery of cloud service's elasticity space boundaries, as a means of learning about the service boundaries and behavior.

\section{REFERENCES}

[1] S. Dustdar, Y. Guo, B. Satzger, and H. L. Truong, "Principles of elastic processes," IEEE Computing, no. 5, pp. 66-71, 2011.

[2] R. Singh, U. Sharma, E. Cecchet, and P. Shenoy, "Autonomic mix-aware provisioning for non-stationary data center workloads," in International Conference on Autonomic Computing, ser. ICAC, 2010, pp. 21-30.

[3] C. Wang, K. Schwan, V. Talwar, G. Eisenhauer, L. Hu, and M. Wolf, "A flexible architecture integrating monitoring and analytics for managing large-scale data centers," in International Conference on Autonomic Computing, ser. ICAC, 2011, pp. 141-150.

[4] S. Meng, A. K. Iyengar, I. Rouvellou, L. Liu, K. Lee, B. Palanisamy, and Y. Tang, "Reliable state monitoring in cloud datacenters," in International Conference on Cloud Computing Technology and Science, ser. CLOUD. IEEE, 2012, pp. 951-958.

[5] G. Katsaros, G. Kousiouris, S. V. Gogouvitis, D. Kyriazis, A. Menychtas, and T. Varvarigou, "A self-adaptive hierarchical monitoring mechanism for clouds," Journal of Systems and Software, vol. 85, no. 5, pp. 1029 1041, 2012. [Online]. Available: http://www.sciencedirect.com/science/ article/pii/S0164121211002998

[6] D. J. Dean, H. Nguyen, and X. Gu, "Ubl: unsupervised behavior learning for predicting performance anomalies in virtualized cloud systems," in International Conference on Autonomic Computing, ser. ICAC. ACM, 2012, pp. 191-200.

[7] L. M. Vaquero, D. Morán, F. Galán, and J. M. Alcaraz-Calero, "Towards runtime reconfiguration of application control policies in the cloud," Journal of Network and Systems Management, vol. 20, no. 4, pp. 489512, Dec. 2012

[8] O. Agmon Ben-Yehuda, M. Ben-Yehuda, A. Schuster, and D. Tsafrir, "Deconstructing amazon ec2 spot instance pricing," in International Conference on Cloud Computing Technology and Science, ser. CloudCom, 2011.

[9] S. Yi, D. Kondo, and A. Andrzejak, "Reducing costs of spot instances via checkpointing in the amazon elastic compute cloud," in International Conference on Cloud Computing, ser. CLOUD, 2010.

[10] J. Shao, H. Wei, Q. Wang, and H. Mei, "A runtime model based monitoring approach for cloud," in International Conference on Cloud Computing, ser. CLOUD, 2010, pp. 313 -320.

[11] M. Dhingra, J. Lakshmi, and S. K. Nandy, "Resource usage monitoring in clouds," in International Conference on Grid Computing, ser. GRID, 2012, pp. 184-191.

[12] S. Clayman, A. Galis, C. Chapman, G. Toffetti, L. Rodero-Merino, L. M. Vaquero, K. Nagin, and B. Rochwerger, "Monitoring service clouds in the future internet," in Future Internet Assembly, 2010, pp. 115-126.

[13] M. Kutare, G. Eisenhauer, C. Wang, K. Schwan, V. Talwar, and M. Wolf, "Monalytics: online monitoring and analytics for managing large scale data centers," in Proceedings of the 7th international conference on Autonomic computing, ser. ICAC, 2010, pp. 141-150.

[14] B. Konig, J. Alcaraz Calero, and J. Kirschnick, "Elastic monitoring framework for cloud infrastructures," IET Communications, vol. 6 , no. 10, pp. 1306-1315, 2012. 